\title{
Fatigue crack growth from handling surface anomalies in a nickel based superalloy at high temperature
}

\author{
Stéphane Gourdin ${ }^{1,2, a}$, Luc Doremus ${ }^{1,2, b}$, Yves $\operatorname{Nadot}^{1, \mathrm{c}}$, Gilbert Hénaff ${ }^{1, \mathrm{~d}}$, and Stéphane Pierret ${ }^{2, \mathrm{e}}$ \\ ${ }^{1}$ Institut Pprime UPR CNRS 3346, Département Physique et Mécanique des Matériaux, ISAE-ENSMA, \\ 86961 Futuroscope Chasseneuil, France \\ 2 Snecma, Etablissement de Villaroche, 77550 Moissy Cramayel, France
}

\begin{abstract}
Aircraft engine manufacturers have to demonstrate that handling surface anomalies in sensitive areas of discs are not critical for in-service life of a component. Currently, the models used consider anomalies as long cracks propagating from the first cycle, which introduces a certain degree of conservatism when calculating the fatigue life of surface flaws. Preliminary studies have shown that the first stages of crack propagation from surface anomalies are responsible for the conservative results. Thus, the aim of the study is to characterize the crack propagation from typical surface anomalies and to establish a new crack growth model, which can account for the micro-propagation stage. To separate the effects of the geometry of the anomalies and the residual stress state after introduction of the surface flaws, two V-type anomalies are studied: scratches and dents. Different studies have shown that the residual stresses beneath the anomalies seem to control the fatigue life of samples exhibiting scratches and dents. In order to monitor the crack micro-propagation, a direct current potential drop technique, coupled with heat tints is used during fatigue tests at elevated temperature. Thermal treatments releasing the residual stresses are also used to decouple the effect of crack morphology and residual stresses.
\end{abstract}

\section{Introduction}

Damage tolerance approaches are used by aerospace industries to demonstrate that components meet the certification requirements in terms of handling damage tolerance. Indeed, during operation or maintenance, rotor discs can be subjected to the introduction of flawtype surface anomalies. Therefore damage tolerance approaches can be used to calculate the fatigue life of a crack propagating from the surface flaw. Currently, such crack propagation models consider surface anomalies as a semi-elliptical long crack propagating from the first cycle. Such approaches often result into conservative crack propagation lives (up to a factor 10 depending on the application stress and temperature) due to the fact that the model does not directly address the phenomena controlling crack initiation and the subsequent micro-propagation stage. Several factors may cause this conservatism: an invalid assumption, which considers the anomaly as a long propagating crack, potential short crack behaviour from the micro-notch formed by the anomaly or geometry and residual stresses effects.

\footnotetext{
a stephane.gourdin@ensma.fr

b luc.doremus@ensma.fr

${ }^{c}$ yves.nadot@ensma.fr

d gilbert.henaff@ensma.fr

e stephane.pierret@snecma.fr
}

It was indeed shown that the fatigue strength of the material is highly influenced by three elements [1]:

- Stress concentration induced by the anomaly geometry: anomalies change the local stress field in the vicinity of the impact sites under external applied load.

- Residual stresses induced by the impact causing the anomaly.

- Anomaly induced micro-cracking or micro-structural distortion responsible for a faster initiation under fatigue loading.

Thus, the aim of the study is to characterise the influence of surface flaws on the propagation in this complex stress field, in order to establish a new crack growth model that can account for the various phenomena observed. Some results of fatigue crack growth tests, which present the influence of residual stresses will be shown as well as fracture surfaces from scanning electron microscopy (SEM). Finally, the modelling strategy used will be outlined.

\section{Experimental procedures}

To evaluate the behaviour of surface anomalies, fatigue crack growth experiments were carried out in load control conditions on a MTS $810( \pm 100 \mathrm{kN}$ capacity $)$ servohydraulic testing machine. Experiments were conducted on specimens with a rectangular cross-section of $3.5 \times$ $8.3 \mathrm{~mm}$ exhibiting two anomalies, one on each face. Before

This is an Open Access article distributed under the terms of the Creative Commons Attribution License 4.0, which permits unrestricted use, distribution, and reproduction in any medium, provided the original work is properly cited. 


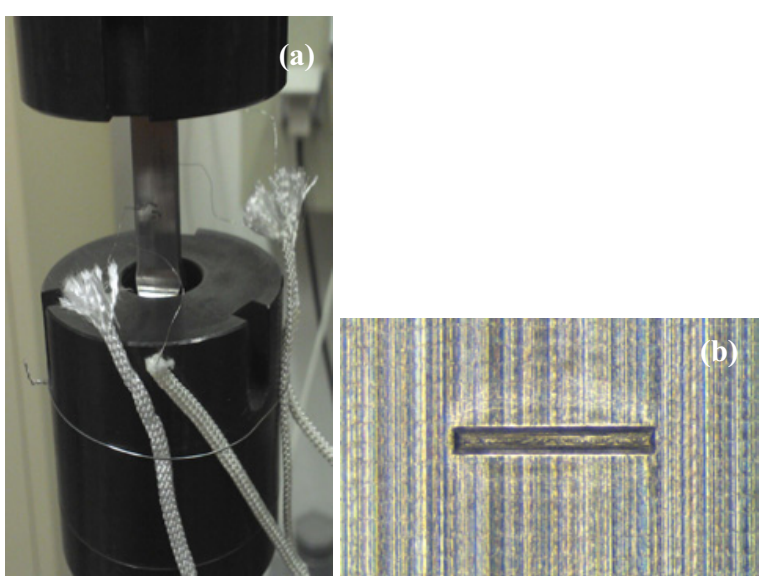

Figure 1. Specimen with platinum wires welded for drop potential measurements (a) and $50 \mu \mathrm{m}$ V-type dent (b).

introducing the anomalies, the specimens were rectified. Two kinds of surface flaws were tested: scratches and dents, both having the same V-type profile, to separate geometry and residual stresses effects, with a depth range from $50 \mu \mathrm{m}$ to $200 \mu \mathrm{m}$. Scratches and dents are the most common surface anomalies observed on discs. The V-type geometry chosen simulate the drop or the scratch of a tool during maintenance operations.

The crack propagation from each anomaly was monitored by the Direct Current Potential Drop (DCPD) technique. For that purpose, $0.1 \mathrm{~mm}$ diameter platinum wires were welded at $500 \mu \mathrm{m}$ from the tip of the scratch or the dent. Heat tints were also used during fatigue tests to correlate potential values and crack size, and also to have information about the crack morphology. The progressive thickening of the oxide layer causes the formation of heat tints. To accentuate these effects, the specimen was kept at high temperature during few hours with no load applied.

Load conditions are determined according to the stress field encountered during in-service operations in lowpressure turbine discs with two maximum stresses: $\sigma_{\max 1}$, $\sigma_{\max 2}\left(\sigma_{\max 1}<\sigma_{\max 2}\right)$, one load ratio $\mathbf{R}_{\sigma}$ with a sinusoidal wave shape at a frequency of $2 \mathrm{~Hz}$. Temperatures covered are between $200^{\circ} \mathrm{C}$ and $700^{\circ} \mathrm{C}$.

The material used in the present study is a nickel based superalloy $\gamma / \gamma^{\prime}$. For confidentiality reasons, it is not possible to provide further details on the designation and the chemical composition of this material; we will name it AlloyX. However some additional results obtained in the nickel based superalloy Inconel 718DA will also be shown.

\section{Results and discussion}

\subsection{Effect of residual stresses on fatigue crack growth}

Fatigue crack growth results corroborate the statements given in the introduction, that experimental fatigue life is higher than calculated fatigue life for all testing parameters. To compute this fatigue life, we consider both scratches and dents as an initial semi-elliptical crack. The extent of the crack along the surface and the interior depth

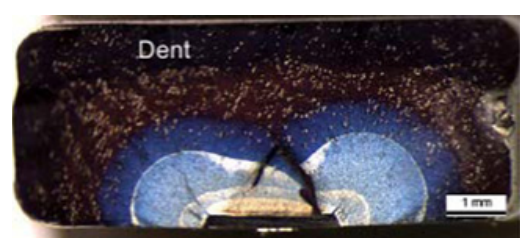

Figure 2. Fracture surface from $200 \mu \mathrm{m}$ dent - Inconel 718DA.

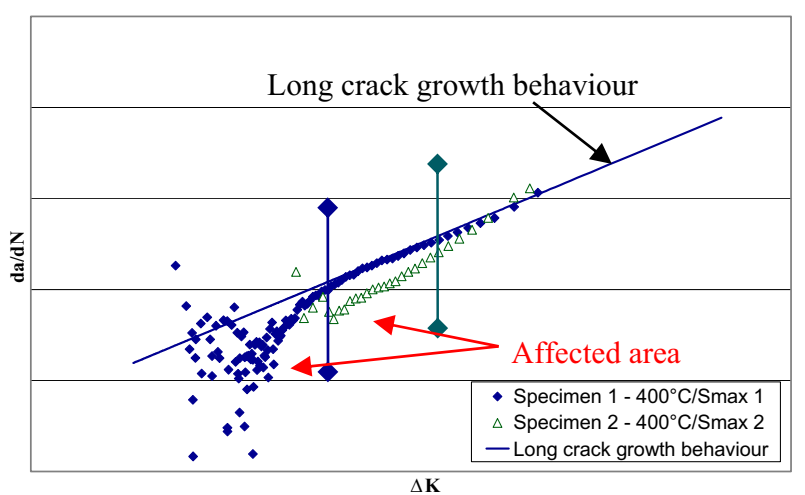

Figure 3. Fatigue crack growth results for $100 \mu \mathrm{m}$ scratches AlloyX.
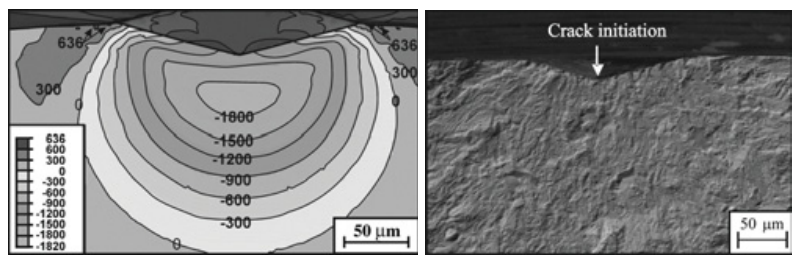

Figure 4. Residual stress field due to Vickers-pyramid indenting and fracture surface of specimen loaded at $770 \mathrm{MPa}-$ Coarsegrain steel [4].

is then calculated using a Paris law. The end of calculation is defined by the crack length when the uncracked area breaks in a ductile manner. Two assumptions can be made: the modelling strategy is not suitable for this application or the material behaviour with an anomaly is different from a smooth specimen.

Heat tints coupled with potential drop values indicate a very low initiation life, less than $30 \%$ of the total life, and a particular crack front morphology (Fig. 2). According to fatigue crack growth rate curves, the fatigue life seems to be controlled by the early stages of crack propagation. A decrease in the crack growth rates is indeed measured over a few hundreds of microns below the anomaly (Fig. 3). Once this affected area is exceeded, the experimental crack growth rate increases to reach the standard calculated crack growth rate of the material. This would indicate that the assumption regarding "long crack" growth behaviour is reasonable.

As previously mentioned, the creation of a surface flaw produces a residual stress state. Several authors measured a compressive residual stress state below dynamic dents [2,3] and also quasi-static dents (Fig. 4) [4], coupled with a tensile residual stress state at the surface. Obviously, those complex stress fields depend on the material, the velocity of the introduction (dynamic or quasi-static) and also the geometry of the indent. Nevertheless, compressive 


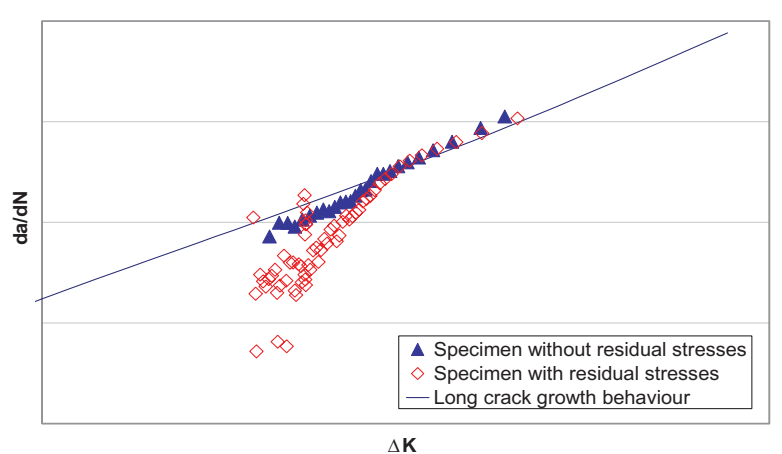

Figure 5. Effect of residual stresses on crack propagation rates Inconel 718DA.

residual stresses under the anomaly could explain the slower crack propagation observed. It has been known for years that compressive stresses may be beneficial in increasing resistance to fatigue failures. This is, for instance, the principle of various surface treatments such as shot peening.

In order to demonstrate the effect of the residual stresses below the surface flaw on the crack propagation rate, fatigue crack growth experiments were carried out on specimens of Inconel 718 DA that exhibit surface flaws after heat treatment to release residual stresses. These experiments also contribute to an improved understanding of the particular crack morphologies observed; the compressive residual stress state is such that internal crack growth is difficult but more favourable for crack extension at corners. In contrast, the crack morphology in the specimen without residual stresses is not affected and shows the expected semi-elliptical shape. These experiments clearly indicate that the experimental (after heat treatment) and calculated crack growth rate are similar (Fig. 5), which sheds light on the influence of the residual stress field beneath the surface flaw on the crack growth rate and the crack morphology.

In spite of the severity of surface anomalies, such as dents, experimental results demonstrate that this kind of surface flaw propagates slower than scratches. A higher compressive residual stress state in the material may be an explanation for this observed phenomenon.

\subsection{SEM analysis}

All specimens tested failed from one of the two surface flaws introduced. In most cases, a crack also initiated from the second surface anomaly. SEM analyses of fracture surfaces reveal a stage II transgranular cracking mode for both materials and for all of the conditions investigated (temperature, loading, depth of anomalies).

Three different areas on the fracture surfaces can be distinguished (Fig. 6):

- Firstly, one can note a relatively smooth region below the anomaly, which may be due to a high distortion of the microstructure (shear bands, distorted or crushed grains). Few fatigue striations, closely spaced, are observed, confirming a slower crack growth (Zone 1).
- In the middle of crack propagation, fatigue striations are much more present and spaced, which corroborate a higher crack growth rate (Zone 2).

- Finally, at the end of crack propagation, a rough and irregular surface showing micro-voids and dimples was found, indicative of ductile fracture (Zone 3).

However, the AlloyX appears to show different behaviour at low $\left(\sigma_{\max 1}\right)$ and at high stress level $\left(\sigma_{\max 2}\right)$. At high applied stress, we observed the nucleation of several secondary micro-cracks maybe due to a high local stress intensity factor. These micro-cracks were formed in a direction normal to the plane of the pre-existing crack and created some marks on the fracture surface (Zone 4). Furthermore, when looking at fractured specimens on a macroscopic scale, a plane (stable) propagation is noticed at low stress level which is not observed at high stress level (Fig. 7). Indeed, the material behaviour does not appear to be homogeneous in the specimen; it seems to be mainly dominated by plane strain conditions near the crack tip and by plane stress conditions on the edges creating shear lips.

Several authors have already studied shear lips in thin sheets $[5,6]$. The appearance of shear lips on the edges depends on several parameters such as: the material, the environment, the frequency, the amplitude loading and the convexity of the front crack. Beyond a certain stress intensity factor in tensile mode the propagation mode change and components of mode II and III appear. The short crack propagation at the free surface as shown in Fig. 8 combined with higher loading amplitude could explain the shear lips observed. We also noticed that the facets are oriented at $\pm 45^{\circ}$ and they could be either in the same direction, or be in opposite directions (Figs. 7b and c). Friction areas are observed just before shear lips, which support potential shear modes II and III (Fig. 9).

\subsection{Modelling of fatigue crack propagation from surface anomalies}

Different crack propagation models from notches exist but few of them take account of residual stress effects below an anomaly. Generally, residual stresses are taken into account by considering them as an additional loading. Indeed, the residual stresses modify the stress intensity factor $K$ and consequently the load ratio $R$. In this way, the total stress intensity factor is the superposition of the stress intensity factor due to loading and that due to the initial pre-existing residual stress field (Eq. (1)). The superposition theory is often used when assessing the effects of a residual stress field on fatigue crack propagation and appears to be the most appropriate solution. On the over hand, the use of superposition considers only the initial residual stress field in the uncracked structure, which carries the assumption that no redistribution or relaxation of residual stresses occurs during crack propagation.

$$
K_{\text {total }}=K_{\text {loading }}+K_{\text {residual stress }} .
$$

For cracks propagating from surface anomalies, the residual stress state is not the only parameter to consider. Indeed Ding et al. have shown that there is a competition between the stress concentration factor $K_{t}$ due to the 

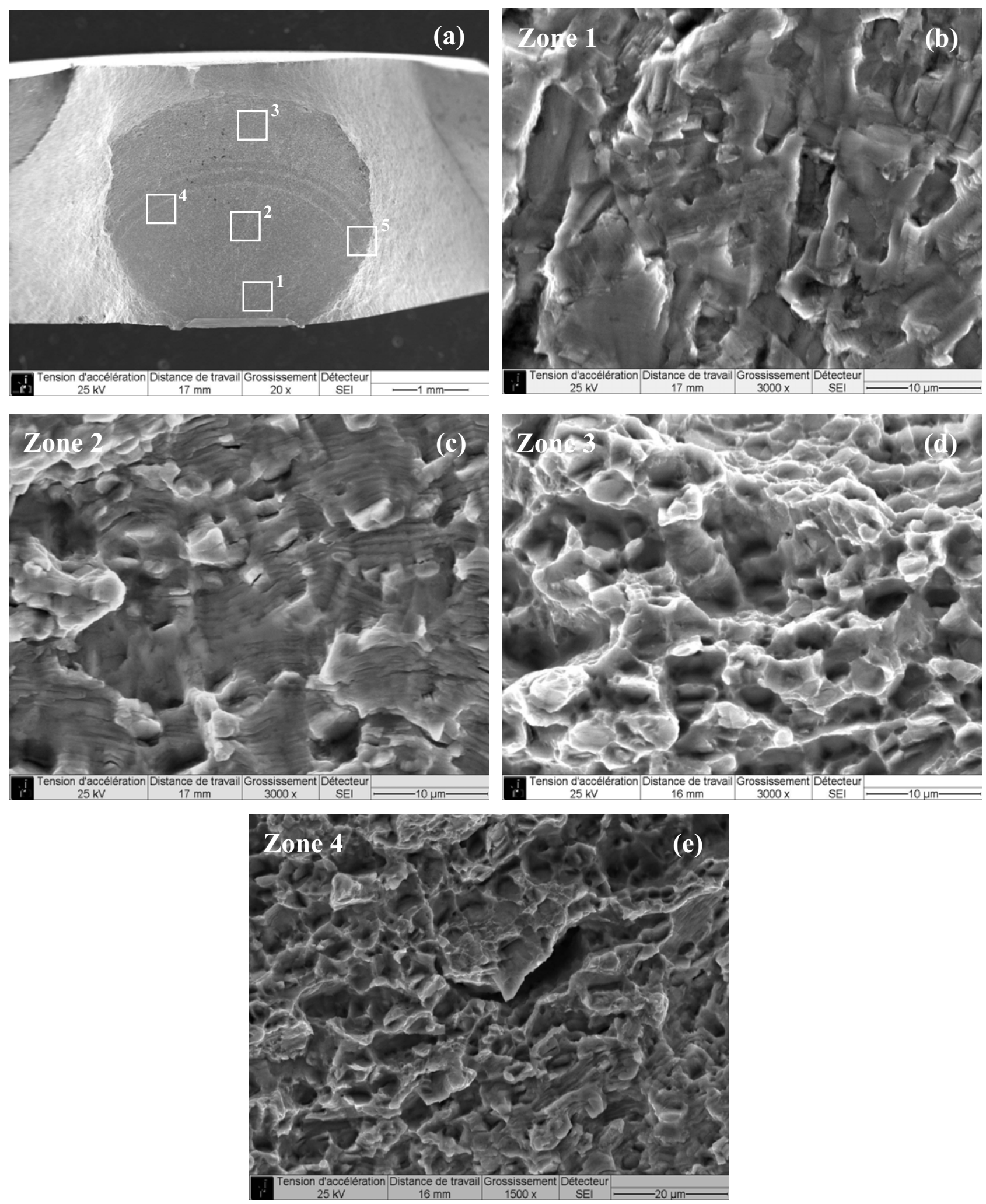

Figure 6. (a) Fracture surface of $100 \mu \mathrm{m}$ scratch $\left(400^{\circ} \mathrm{C} / \sigma_{\max 2}-\right.$ AlloyX), (b-e) secondary electron images showing the different stages of crack propagation. 

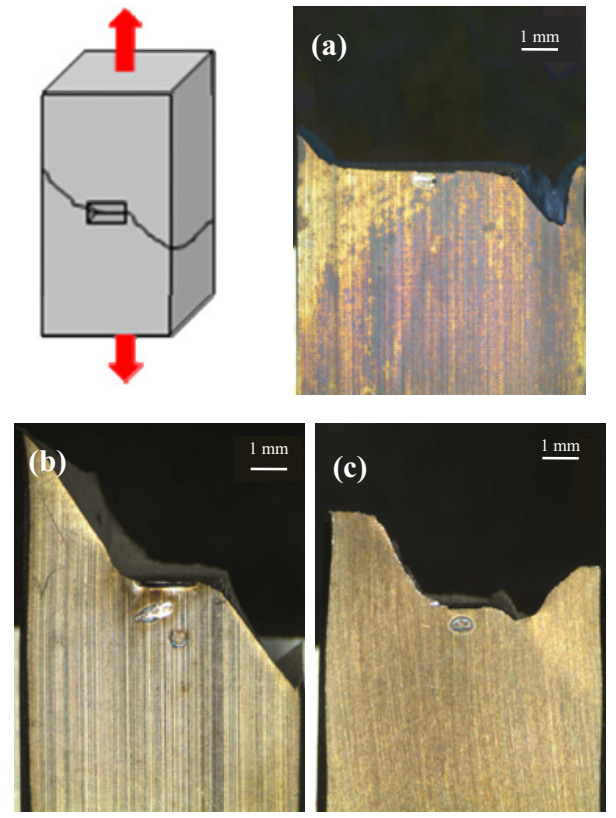

Figure 7. (a) Plane propagation at low stress level, (b) opposite shear lips (c) and shear lips in the same direction at high stress level - AlloyX.

anomaly geometry and the residual stress level [7]. The stress concentration factor peaks near the anomaly while the residual stresses have very little influence. Conversely, away from the surface flaw, the $K_{t}$ effect decreases while the residual stress effect is higher (maximum of compressive stresses). Therefore, these two factors are necessary to model the crack growth from surface anomalies. The modelling strategy set up is divided into three steps:

- Numerical simulations of the introduction of a surface anomaly (scratch or dent) are carried out in order to obtain the residual stress field.

- Then, numerical simulations of the crack propagation in this complex field are computed for all testing parameters. The stress intensity factor is then calculated along the entire front crack and at every increment.

- Finally, the specimen fatigue life is calculated with a Paris law, knowing the stress intensity factor and the crack depth.

Preliminary studies have been made to demonstrate the feasibility of this strategy. Numerical simulations of a dent have been carried out. Then, the stress field (superposition of residual stresses and stress due to loading) is given as a sum of monomials such as $n+m<4$ (Eq. (2)). A semi-elliptical crack is assumed and the crack growth is then calculated using a Paris law along the entire crack front. The Fig. 10 show the qualitative results obtained. This strategy permit to find the crack front morphology observed experimentally and consequently to demonstrate the influence of the residual stresses on crack propagation. Indeed, the internal crack propagation is slowed down and faster at corners as expected.

$$
\sigma_{z z}(x, y)=\sigma_{0} \cdot(x / c)^{n} \cdot(y / a)^{m} .
$$

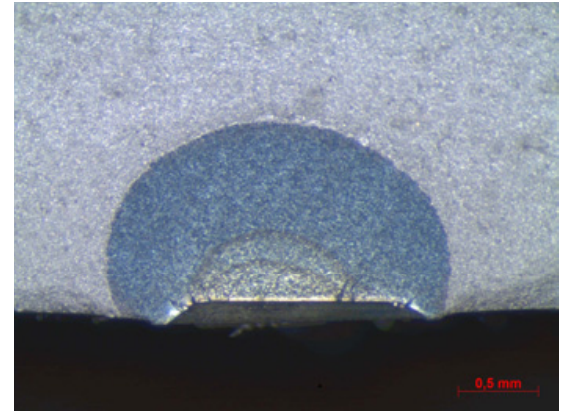

Figure 8. Fracture surface of the second anomaly, which did not lead to specimen failure; observation of short propagation at the free surface - AlloyX.

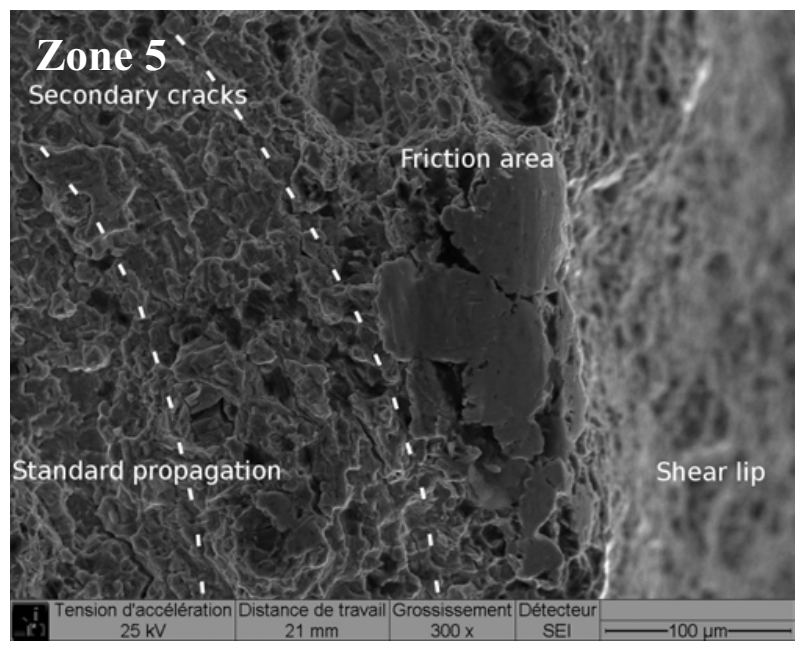

Figure 9. Fracture surface of $100 \mu \mathrm{m}$ scratch showing a shear lip $\left(400^{\circ} \mathrm{C} / \sigma_{\max 2}-\right.$ AlloyX).
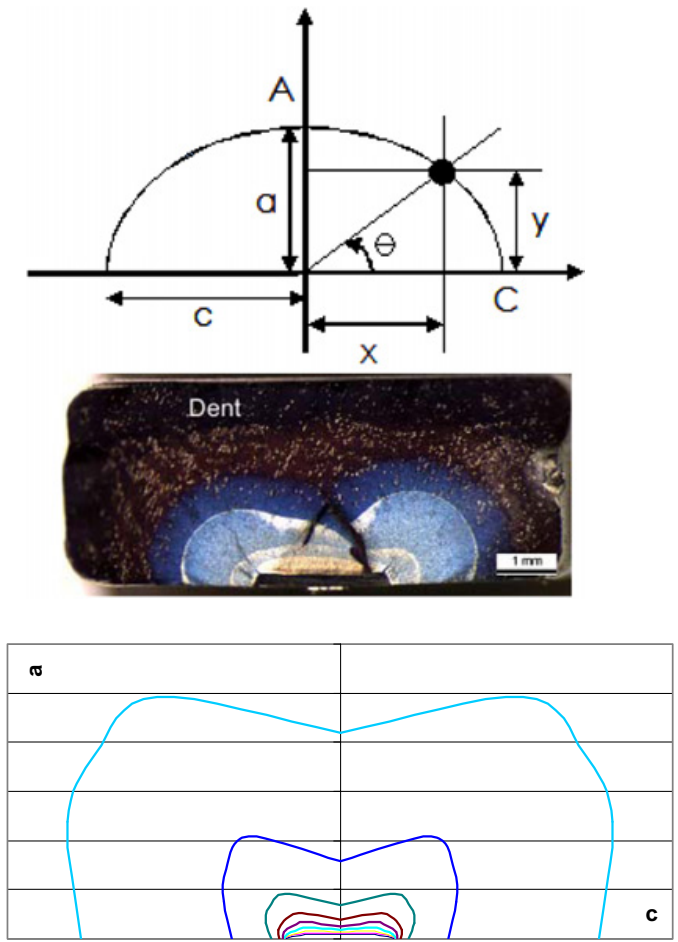

Figure 10. Fracture surface from $200 \mu \mathrm{m}$ dent - Inconel 718DA and crack front morphology calculated. 
The strategy set up should permit the prediction of less conservative and more accurate fatigue lives. Nevertheless, an experimental evaluation using X-ray diffraction or micro-hardness will have to be performed to compare with numerical simulations in order to validate our simulation methodology. Moreover, this strategy set up at specimens scale, will have to be applicable to disc-scale so that it could be used for dimensioning.

\section{References}

[1] X. Chen, J.W. Hutchinson, J. Mech. Phys. Solids, 50, 2669-2690, (2002)
[2] B.L. Boyce, X. Chen, J.W. Hutchinson, R.O. Ritchie, Mech. Mater., 33, 441-454, (2001)

[3] P.G. Frankel, P.J. Withers, M. Preuss, H.T. Wang, J. Tong, D. Rugg, Mech. Mater., 55, 130-145, (2012)

[4] T. Vuherer, L. Milović, V. Gliha, Int. J. Fatigue, 33, 1505-1513, (2011)

[5] J. Schijve, Eng. Fract. Mech., 14, 789-800, (1981)

[6] K.D. Thompson, S.D. Sheppart, Eng. Fract. Mech., 43, 73-82, (1982)

[7] J. Ding, R.F. Hall, J. Byrne, J. Tong, Int. J. Fatigue, 29, 1350-1358, (2007) 